\title{
Visitor Perceptions of the Potential for Virtual Reality in a Small Museum
}

\author{
Eric Mc Namara \\ Dept. of Information Technology \\ Limerick Institute of Technology \\ eric.mcnamara@lit.ie
}

\author{
Dr Séamus Ó Ciardhuáin \\ Dept. of Information Technology \\ Limerick Institute of Technology \\ seamus.ociardhuain@lit.ie
}

\author{
Denise Mc Evoy \\ Dept. of Design \& Visual Art \\ IADT Dún Laoghaire \\ denise.mcevoy@iadt.ie
}

\begin{abstract}
Virtual reality offers museums the possibility of creating immersive and interactive user experiences which have not hitherto been possible. It is essential, however, to take into consideration the views, attitudes and experiences of the visitors to museums to achieve the maximum impact of these technologies. We present the results of a survey of visitors to a small museum, undertaken as part of the design work for a VR installation. The results show a good awareness of virtual reality among the museum's visitors and some experience with it, as well as a clear interest in it as a way of enhancing the museum experience.
\end{abstract}

Virtual Reality, Immersive Technologies, Museums.

\section{INTRODUCTION}

Museums wish to present their exhibits in a more attractive and exciting manner to attract visitors. Recent surveys in Europe show that about $35 \%$ of museums have already started developments with some form of three-dimensional (3D) presentation of objects (Tsapatori et al., 2003). Many researchers have considered museum settings as a potential application domain for mixed reality entertainment while also attempting to produce public experiences of genuine cultural value (Bowers et al., 1996; Caulton, 1998; Fraser et al., 2003; Grinter et al., 2002). It is evident that museums are starting to recognise the potential offered by immersive technologies. The decreasing cost and improved availability of virtual reality (VR) makes it an attractive way for small museums to increase the scope of exhibits.

The Frank McCourt Museum is a small privatelyowned museum in Limerick, Ireland. It combines conventional and interactive exhibits relating to the life and work of Frank McCourt. Located in the building where McCourt attended school, a notable feature of the museum is the reconstruction of a 1930s school classroom which visitors can explore. There are also two reconstructed rooms, based on descriptions in his books and interviews with the author as well as many contemporary artifacts and extensive archives.

Working in collaboration with the Frank McCourt Museum, the Frank's City project aims to create an immersive experience relating to life in Limerick over the last century. Vanished aspects of the city will be brought to life based on the experiences and memories of the author Frank McCourt (1930-2009), best known for Angela's Ashes, his memoir of growing up in Limerick in the 1930s and 1940s for which he received the Pulitzer Prize in 1997. A central project aim is to allow users to engage with the past in an entertaining and informative way using VR to enhance the physical exhibits, and by recreating aspects of the city which have been lost.

As a first step in the project, visitors to the museum were surveyed, identifying the demographic features of the visitors, their views and attitudes to VR technology in general, experience (if any) with VR, and inquiring how they thought VR might beneficially be used in a museum context to improve their experience.

\section{LITERATURE REVIEW}

The use of immersive Virtual Reality technology is not a new trend. It has been used by the military and industrial research and development communities for over a decade.

However, as this technology develops, research is expanding from the military and scientific fields into more multidisciplinary areas, such as construction, culture, art, education, and humanities. Furthermore, cultural centres and museums may position themselves better by utilising virtual reality technologies. 
Museum exhibits typically incorporate attentiongetting tactics to lure visitors over to exhibit elements, so visitors can take away that exhibits message from their experience. While new technologies may be part of the answer, the museums and exhibits desired message being delivered by technologies cannot lack material or be empty. Should this happen the technology being used would simply become a gimmick as the museum and each exhibit "message" is the most effective, engaging and appropriate method of communication and is what defines them. However, introducing new technologies is, of course, one way to reach visitors but even the best technology has limitations.

An important issue is whether the public will use and interact with these emerging environments. Museums may not directly resolve these questions and issues they currently face but, by providing imaginative and intelligent opportunities, they can play a part in the development and assessment of interactive technologies. Museum exhibits, where high-quality image technology is required, can become one of the most promising applications for virtual reality technology. Also, it may reach a segment of people and a wider audience who are not usually museum-goers. As museums show more interest in virtual reality how ready are museums for disruptive shifts in visitor behaviour and changes and what sort of services would museums be able to provide in their environment. When used for cultural heritage purposes, a virtual reality exhibit may give scholars a completely new way of communicating the scientific results of the archaeological investigation within the scientific community and improving the way in which these results are communicated to the public (Niccolucci, 1999).

Virtual Reality is one of these technologies that can have a wide impact on many areas including cultural heritage which is not commonly associated with computer technologies (Tan et al., 2013). In this respect, Virtual Reality can offer solutions by using visualisation of 3D modelling technology. By recreating and documenting cultural artefacts or environments digitally not only are you affording visitors to interact with them but you're also saving a piece of history electronically like "a visual preservation of old memories".

This study is using design-based research methodology (DBR). In the early 1990s, DBR was proposed by Brown (1992) and Collins (1992) as an approach that covers current methods and addresses the issue of linking theory and practice in educational research. Since then, DBR has evolved and has been used in learning research in various forms (Confrey, 2006). DBR involves the creation of a theoretically-inspired innovation, usually a learning environment, to directly address a local problem (Barab, 2006). In recent years, this approach has been the subject of extensive discussions and reflections (Barab \& Squire, 2004; Kelly, 2003; Kelly, Lesh, \& Baek, 2008; Sandoval \& Bell, 2004). The goal of DBR is to use the close study of a single learning environment, usually as it passes through multiple iterations and as it occurs in naturalistic contexts, to develop new theories, artefacts, and practices that can be generalised to other schools and classrooms.

\section{SURVEY METHODOLOGY}

In this study, a survey form containing 24 questions was used, and semi-structured individual interviews were undertaken with visitors. Twenty visitors to the museum were surveyed during the months of January and February 2018. In this paper, we focus on the results relating specifically to virtual reality; there were also questions relating to the museum's existing exhibits. The interview data were input to Survey Monkey (Survey Monkey Inc, 2018) for analysis and visual presentation. The questionnaire may be seen online at https://www.surveymonkey.com/r/MR97NRB

For this survey, no specific age group was selected, but all interviewees were aged 18 years or over. All interviewees had printed consent forms to sign and were informed that this was a non-commercial educational project and that all personal information was anonymous and never stored. Ethical clearance for the project was obtained in accordance with the sponsoring institute's policies.

\section{RESULTS}

The age profile of the twenty interviewees is shown in Figure 1 (survey question 1). 60\% of those interviewed said that they had read Angela's Ashes, indicating an awareness of the museum's subject matter and an established interest in it, rather than simply casual visitors (survey question 3). A similar percentage had visited other museums in the preceding year (survey question 4). 


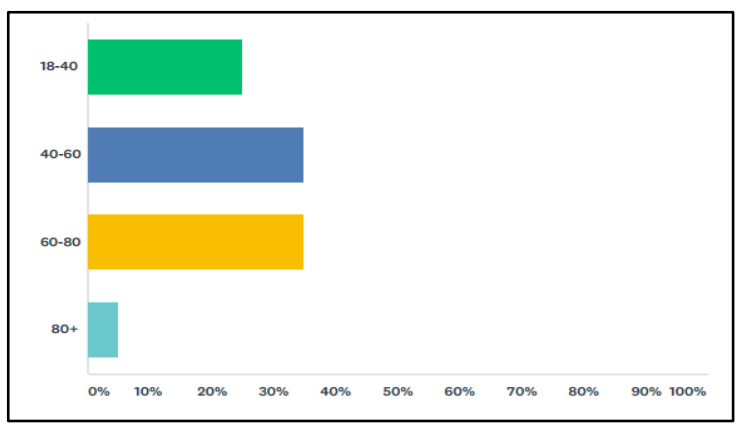

Figure 1: The age profile of the interview sample.

Visitors were then asked how they thought museums should provide access to archives and libraries, with a choice between "physical" (i.e. being able to access the objects themselves), "digital interactive" access, or both (survey question 9). The results are shown in Figure 2. The responses show a clear preference for both electronic and physical presentation of museum content.

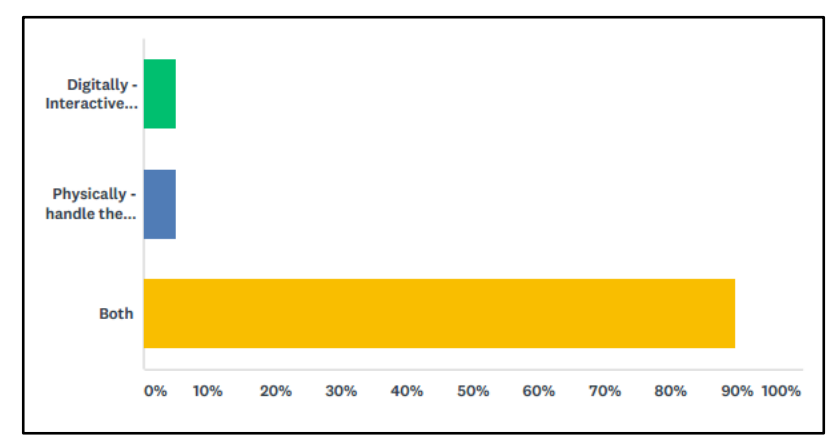

Figure 2: Interviewees' preferences for access methods to museum archives and libraries.

Interviewees' familiarity with, and interest in, virtual reality was assessed in several questions. It was notable that all expressed an interest in the idea of using VR (survey question 20) and $90 \%$ had some familiarity with the concept of VR (survey question 19). $55 \%$ had tried VR at some time (survey question 21), and $90 \%$ expected or planned to use VR (survey question 24).

When asked about their concerns relating to VR (survey question 23), the most common response $(65 \%)$ was lack of knowledge about VR. Other concerns were cost, usability and the possibility of motion sickness (Figure 3). However, 45\% each reported "good" or "great" experience with VR, and only $10 \%$ a bad experience (survey question 22 ).

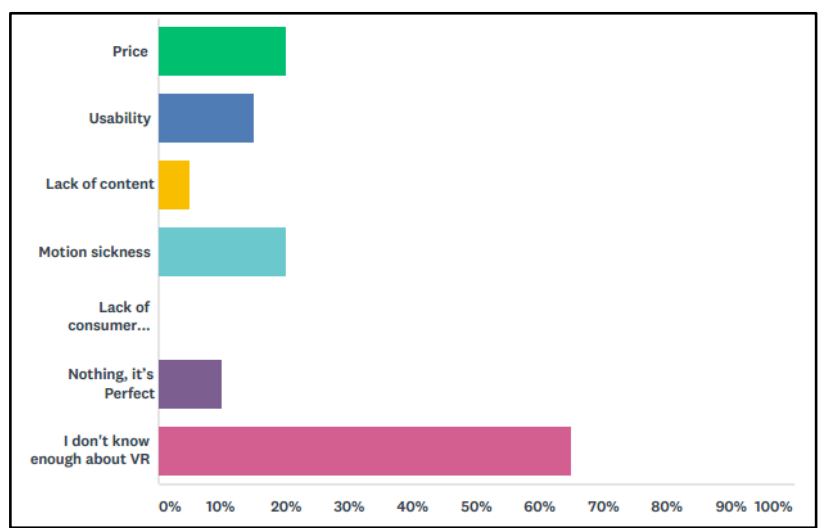

Figure 3: Interviewee concerns relating to Virtual Reality.

Turning to the Frank McCourt Museum, in particular, interviewees were asked about their preferred exhibits (survey question 14). By far the most popular exhibit was the reconstructed 1930s school classroom, which is the most interactive aspect of the museum. This was followed by the two reconstructed rooms which can be viewed but not entered (Figure 4).

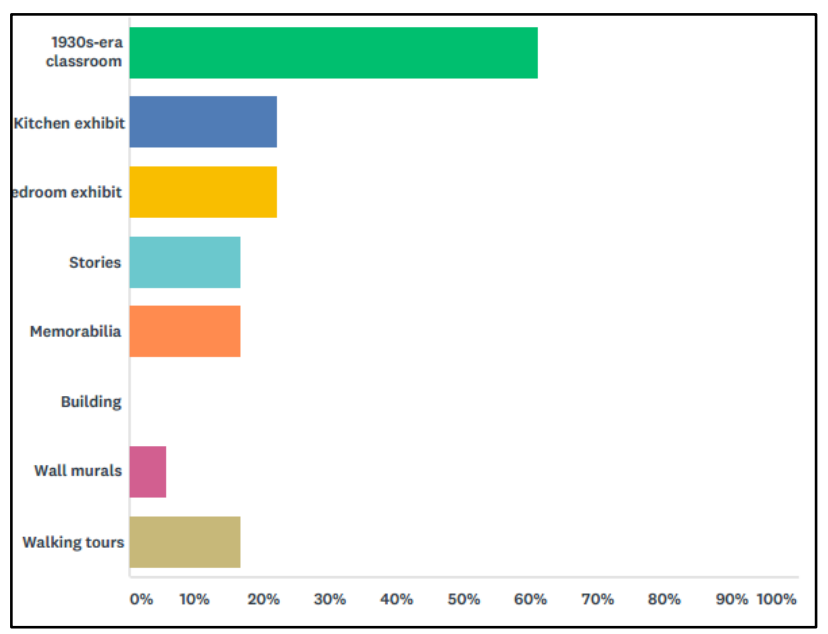

Figure 4: Interviewees' preferred exhibits in the Frank McCourt Museum.

Finally, interviewees were asked to suggest what new type of presentation they would like to see (survey question 18, Figure 5). VR received a strong response here with $40 \%$ selecting it. Interestingly, only $6 \%$ selected augmented reality, with most opting for audio-visual and video exhibits (99\% combined). 


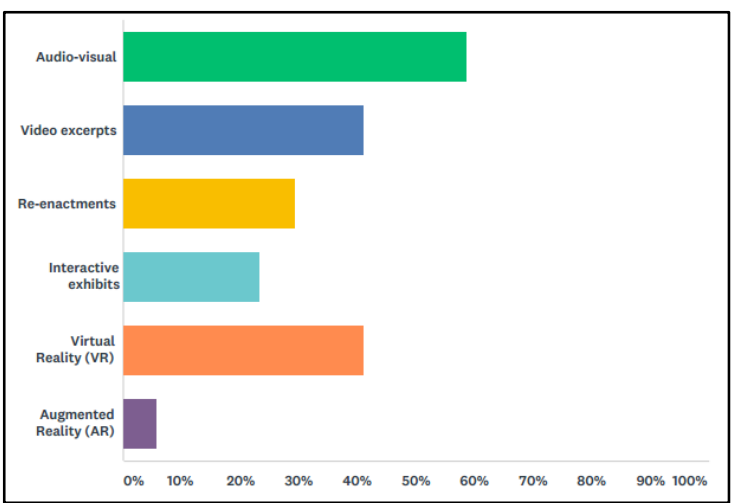

Figure 5: Interviewees' new ways of presentation that are not currently used within this Museum. (More than one item could be chosen, so totals do not sum to $100 \%)$.

\section{DISCUSSION}

The survey results have given some interesting insights into the attitudes of visitors to the Frank McCourt museum in relation to VR and technologies for museum exhibits.

We feel that the level of interest and experience shown is particularly interesting given the broad spread of ages in the sample. The interviewees' concern about their lack of knowledge relating to VR suggests that they feel they should know more because it will be important to them in future. These responses indicate that marketing and industry focus on VR has created broad awareness of the technology, beyond the obvious technicallyliterate audiences such as gamers.

The positive views on the quality of VR experience are notable given the limited availability of very high-quality VR technology as yet. Many users have experienced VR through simple low-resolution devices such as phones rather than the sophisticated installations that are to be expected in a museum environment, where dedicated headsets and computers could be installed. This is encouraging for our research in that museum visitors can be expected to have an even more favourable response to high-quality VR of the type we propose to deploy.

The importance of interactivity is made clear by Figure 4. The opportunity to be present in the classroom environment made it stand out as part of the visitor experience, much more so than the reconstructed rooms in the museum even though they are such a central part of McCourt's book and incorporate many period objects to bring the descriptions to life. However, they lack any interactive component. Virtual reality offers us the potential to create interactive experiences which would not otherwise be possible, for example by making an interactive VR room, or by recreating a street scene of the time - something that would be beyond the resources of a small museum to do in any other way.

The low level of interest shown in augmented reality is curious given the high interest in VR. This may simply be attributable to lack of awareness and experience but is a point to investigate further since on the face of it AR should also have potential in this type of environment.

Our survey did not consider the levels of realism and interaction experienced by visitors and their responses to them. This will be assessed as part of the evaluation of the prototype VR experience under development in the project Museums may not directly resolve these questions and issues but, by providing imaginative and intelligent opportunities, they can play a part in the development and assessment of interactive technologies.

In summary, we found a strongly favourable attitude to the idea of providing VR experiences in museums, indicating that the continuing deployment of VR in the museum environment will be well received by future visitors. Museum exhibits, where high-quality image technology is required, can become one of the most promising applications for VR technology. Public environments such as museums should consider the educational and recreation potential of this medium while examining how they could stay relevant to the culture they serve.

\section{REFERENCES}

Barab, S. (2006). Design-Based Research: A Methodological Toolkit for the Learning Scientist. Cambridge University Press.

Barab, S., \& Squire, K. (2004). Design-based research Putting a stake in the ground. THE JOURNAL OF THE LEARNING SCIENCES, 13(1), 1-14, 47(4), 552-560. https://doi.org/10.1016/j.yjmcc.2009.07.018

Bowers, J., O'Brien, J., \& Pycock, J. (1996). Practically Accomplishing Immersion : Cooperation in and for Virtual Environments. Computer Supported Cooperative Work, 380389. https://doi.org/10.1145/240080.240349

Brown, A. (1992). Design experiments Theoretical and methodological challenges in creating 
complex interventions. Universidade de Aveiro.

https://doi.org/10.1145/1165387.275623

Caulton, T. (1998). Hands-on exhibitions: managing interactive museums and science centres. Psychology Press.

Collins, A. (1992). Towards a design science of education. New Directions in Educational Technology, 15-22. https://doi.org/citeulikearticle-id: 4545332

Confrey, J. (2006). The evolution of design studies as methodology. na.

Fraser, M., Stanton, D., Ng, K. H., Benford, S., O'Malley, C., Bowers, J., ... Hindmarsh, J. (2003). Assembling History: Achieving Coherent Experiences with Diverse Technologies. ECSCW 2003: Proceedings of the Eighth European Conference on Computer Supported Cooperative Work 1418 September 2003, Helsinki, Finland, (September), 179-198. https://doi.org/10.1007/978-94-010-0068-0_10

Grinter, R. E., Grinter, R. E., Aoki, P. M., Aoki, P. M., Szymanski, M. H., Szymanski, M. H., ... Hurst, a. (2002). Revisiting the visit: Understanding How Technology Can Shape the Museum Visit. Proceedings of the 2002 ACM Conference on Computer Supported Cooperative Work, 146-155. https://doi.org/10.1145/587078.587100

Kelly, A. E. (2003). Theme issue: The role of design in educational research. Educational Researcher, 32(1), 3-4.
Kelly, A. E., Lesh, R. A., \& Baek, J. Y. (2008). Handbook of design research methods in education: Innovations in science, technology, engineering, and mathematics learning and teaching. Routledge.

Niccolucci, F. (1999). Virtual Reality in Archaeology: a useful tool or a dreadful toy? Mediaterra Art \& Technology Festival, 99.

Sandoval, W. A., \& Bell, P. (2004). Design-based research methods for studying learning in context: Introduction. Educational Psychologist, 39(4), 199-201. https://doi.org/10.1207/s15326985ep3904_1

SurveyMonkey. (2018). SurveyMonkey: The World's Most Popular Free Online Survey Tool. Retrieved February 27, 2018, from https://www.surveymonkey.com/

Tan, B.-K., Rahaman, H., Durand, E., Merienne, F., Pere, C., Callet, P., ... Avram, G. (2013). Interpreting Digital Heritage: A Conceptual Model with End-Users' Perspective. Journal on Computing and Cultural Heritage, 1(212), 610-622. https://doi.org/10.1145/2072298.2072477

Tsapatori, M., et al. (2003). ORION Research Roadmap for the European archaeological museums' sector (Final Edition). June 2003. 